\title{
Impact of interface orientation on magnetic coupling in highly ordered systems: A case study of the low-indexed $\mathrm{Fe}_{3} \mathrm{O}_{4} / \mathrm{NiO}$ interfaces
}

\author{
I. P. Krug,${ }^{1, *}$ F. U. Hillebrecht, ${ }^{1, \dagger}$ M. W. Haverkort, ${ }^{2,+}$ A. Tanaka, ${ }^{3}$ L. H. Tjeng, ${ }^{2}$ H. Gomonay, ${ }^{4}$ A. Fraile-Rodríguez, ${ }^{5}$ \\ F. Nolting, ${ }^{5}$ S. Cramm, ${ }^{1}$ and C. M. Schneider ${ }^{1}$ \\ ${ }^{1}$ Institut für Festkörperforschung (IFF-9), Forschungszentrum Jülich GmbH, 52425 Jülich, Germany \\ ${ }^{2}$ Physikalisches Institut II, Universität zu Köln, 50937 Köln, Germany \\ ${ }^{3}$ Department of Quantum Matter, ADSM, Hiroshima University, Higashi-Hiroshima, 739-8530, Japan \\ ${ }^{4}$ Bogolyubov Institute for Theoretical Physics, 14-b Metrologichna Street, 03680, Kiev, Ukraine \\ ${ }^{5}$ Swiss Light Source, Paul Scherrer Institute, 5232 Villigen, Switzerland
}

(Received 31 October 2007; revised manuscript received 21 July 2008; published 28 August 2008)

\begin{abstract}
We investigated the influence of the interface orientation on the magnetic coupling for the (110), (001), and (111) interfaces of epitaxial $\mathrm{NiO}$ thin films on single-crystal $\mathrm{Fe}_{3} \mathrm{O}_{4}$. In our analysis, we combine magnetic $\mathrm{x}$-ray spectroscopies with circularly and linearly polarized light, i.e., x-ray magnetic circular and linear dichroism (XMCD and XMLD). A full treatment of the anisotropic XMLD in single-crystalline materials backed by theoretical support allows for a reliable vectorial magnetometry. Both the (110) and the (111) interfaces produce collinear coupling, which at first glance contradicts Koon's theory, while at the (001) interface, a perpendicular (spin-flop) coupling between a ferrimagnet and an antiferromagnet is observed. Furthermore, we find a pronounced enhancement of the interfacial uncompensated magnetization for the (110) interface only, while for the two other interfaces this value is considerably lower. These apparent discrepancies are resolved in terms of a detailed consideration of exchange and magnetoelastic effects for the individual orientations. The results highlight the fundamental role of the crystalline interface orientation in magnetic coupling phenomena involving oxidic systems.
\end{abstract}

DOI: 10.1103/PhysRevB.78.064427

PACS number(s): 75.70.Cn, 75.70.Ak, 75.25.+z

\section{INTRODUCTION}

Two distinct magnetic materials in direct contact will experience a strong coupling across the interface mediated through short-ranged exchange interactions. This magnetic coupling causes a change in the magnetic properties of the individual constituents - a complex situation, which is sometimes termed "proximity effect." The latter can have different facets reflected in a change of the magnetic anisotropy, the magnetic moments, the local spin configuration, or the magnetic ordering temperature. The interaction between a ferromagnet (FM) or a ferrimagnet (FIM) and an antiferromagnet (AF) gives rise to a very peculiar proximity effect, which is known as exchange bias and exhibits all of the features mentioned above. Discovered in the 1950s by Meiklejohn and Bean, ${ }^{1}$ it has in the meantime become a key ingredient for the fabrication of high-density hard disks, magnetic-field sensors, and more sophisticated spintronic devices. ${ }^{2,3}$ Despite its technological relevance, the effect is not yet entirely understood in terms of the microscopic mechanisms that give rise to it. A major obstacle on the way to a better comprehension is in fact posed by the nature of an antiferromagnet, which-due to its vanishing net moment-is directly accessible only by a few techniques, unfortunately most of them requiring bulk samples (such as neutron diffraction, ${ }^{4}$ for example). Even fewer techniques exist that provide the capability to differentiate between the bulk and the surface or interfacial magnetic structures, most of them in the fields of $\mathrm{X}$-ray diffraction ${ }^{5-7}$ and spectro(micro)scopy. ${ }^{8-12}$

A central aspect of the magnetic proximity effect is the crystallographic structure of the boundary region between the two materials, which may influence the interfacial coupling in two ways:

(1) Electronic effects will arise due to the breaking of translational symmetry such as, for example, an anisotropic exchange interaction, altered crystal-field symmetry, and electronic hybridization across the interface. Those effects will be generally short ranged, particularly in the poorly conducting oxidic materials treated in our study. Thus, the purely electronic proximity effects are generally restricted to a very narrow region of a few atomic layers on either side of the interface (see, for example, Ref. 13 and references therein).

(2) In the case of an epitaxial lattice mismatch, magnetoelastic effects will generally occur, which depend on the orientation of the planar epitaxial strain with respect to the crystal lattice. In contrast to the short-range electronic interactions at the interface, the strain-induced effects will extend farther into the sample. For tetragonally strained $\mathrm{NiO}$ in the two systems $\operatorname{Ag}(001) / \mathrm{NiO}(c / a>1)$ (Ref. 14) and $\mathrm{MgO}(001) / \mathrm{NiO}(c / a<1),{ }^{15}$ the strain will result in a preferred spin arrangement, which is in plane for $c / a>1$ and out of plane for $c / a<1$. This effect was also predicted theoretically by calculations of the pseudodipolar anisotropy energy of NiO. ${ }^{16}$ For strained $\mathrm{CoO}(001)$ layers, experiments resulted in a similar outcome: The $\mathrm{CoO}$ spin axis always follows the tetragonal compression; i.e., for $c / a>1$, the spin axis is in plane and for $c / a<1$ it is out of plane. ${ }^{17}$ The mechanisms for this behavior, however, are essentially different between both materials since for $\mathrm{CoO}$ the orbital degrees of freedom are the driving force for magnetoelasticity, ${ }^{17}$ while for $\mathrm{NiO}$ the main mechanism is exchange striction. ${ }^{18}$ 
The importance of the crystallographic interface orientation was pointed out for the first time by Moran et al. ${ }^{19}$ and Nogues et al., ${ }^{20}$ who observed a dependence of the exchange bias field $H_{\mathrm{eb}}$ on the crystallographic orientation in singlecrystalline $\mathrm{FeF}_{2}-\mathrm{Fe}$ systems. In their study, magnetoelastic effects were considered to contribute to the overall magnetic behavior, but their influence was not discussed in much detail. In a theoretical argument Qian and Hubner ${ }^{21}$ predicted the orientation-dependent crystal-field splitting to have an important effect on the electronic and magnetic properties of the interface region. Despite extensive theoretical work on the proximity effect at interfaces (see, for example, Ref. 13 and references therein), experimental studies are still scarce, partly because only few techniques are able to investigate buried interfaces with sufficiently high selectivity and/or depth resolution.

In this paper we address the question of how the crystallographic orientation influences the FM/AF magnetic proximity effect via exchange coupling and magnetoelastic effects in the specific system $\mathrm{NiO}$ on $\mathrm{Fe}_{3} \mathrm{O}_{4}$. We can distinguish two basic coupling geometries in FM/AF systems, namely, collinear and spin-flop couplings. In the latter configuration, the AF spin axis orients by $90^{\circ}$ with respect to the FM.

We found that the formation of either one of the two geometries cannot be satisfactorily predicted by solely applying the criteria given in the model of Koon. ${ }^{22}$ The latter requires a compensated interface for spin-flop coupling, i.e., a balance of spin-up and spin-down states at the interface, resulting in a negligible net magnetic moment. Due to structural arguments, ${ }^{23}$ this condition is practically never fulfilled in the $\mathrm{Fe}_{3} \mathrm{O}_{4} / \mathrm{NiO}$ system, implying that spin-flop coupling should not occur according to Koon's theory. Experimentally, however, we find this type of coupling for the (001) interface, while in the other orientations collinear coupling is observed. This clearly shows that mechanisms beyond interfacial exchange interaction have to be taken into account, such as, for example, magnetoelasticity. The latter is well known to have a significant effect on the magnetic anisotropy in ferromagnetic thin films due to lattice mismatch between the substrate and film and the resulting strain. ${ }^{24}$ To our knowledge, the role of magnetoelasticity in coupling phenomena has been underestimated so far. Certainly more work is needed to quantify its influence in systems with strong directional exchange coupling mechanisms, such as the transition-metal oxides.

Reliable conclusions on this issue, however, are strongly facilitated in the case of highly ordered systems. Thus, we decided to investigate a well-ordered epitaxial system of low lattice mismatch, namely, $\mathrm{Fe}_{3} \mathrm{O}_{4} / \mathrm{NiO}$. This ensures a good crystalline quality of the films by keeping the epitaxial strain and defect density low. Since both constituents are stable oxidic phases, interfacial redox reactions are expected to be minimized, thereby leading to atomically sharp interfaces ${ }^{25,26}$ and defect densities lower than in comparable oxide/metal interfaces. In the latter, a gradual disorder transition zone from oxide to metal may form ${ }^{6,27}$ if the interface cannot be passivated prior to deposition (see, e.g., Ref. 28). In addition, the strongly directional short-range character of the magnetic interactions (double exchange and superexchange) provides a pathway to interpret the crystalline influence on the magnetic structure in a somewhat simpler picture as compared to a more complicated band-structure approach for metals.

In our experiments we employed photoemission electron microscopy (PEEM) in combination with polarized soft $x$ rays to selectively study the magnetic structure at the Fe and $\mathrm{Ni}$ edges by X-ray magnetic circular and linear dichroism (XMCD and XMLD). In this way we are able to distinguish between the two materials and can also selectively probe the buried interface. The use of XMLD especially enables us to extract specific information about the antiferromagnet. Since our films are single crystalline, we explicitly applied the recently developed formalism for the description of anisotropic XMLD in a cubic crystal field, ${ }^{29-33}$ which depends on both the orientations of the spin $\mathbf{S}$ and the polarization $\mathbf{E}$ of the photon field relative to the cubic basis frame. In contrast to previous reports, where the influence of the crystalline symmetry was generally neglected, $8,9,11,15,34$ we can now reliably determine the spin-axis orientation from our microspectra.

The paper is structured as follows: In Sec. II, we will describe the method of sample preparation. In Sec. III we will give an introduction into the evaluation method using the theoretical framework for anisotropic XMLD. In Secs. IV A-IV C we will present the experimental data of the three interfaces and describe the analysis of the coupling character. In Sec. IV D we will quantitatively compare the uncompensated magnetization component arising at the three interfaces due to the interfacial exchange interaction and possible crystalline phase reconstructions. Finally, we will discuss the results in Sec. V and summarize our conclusions in Sec. VI.

\section{SAMPLE PREPARATION}

All our samples were prepared in situ and measured directly after preparation to avoid the necessity of capping and to be able to study the as-grown magnetic structure. We used synthetic magnetite single crystals polished to the chosen crystallographic orientation to typically better than $0.5^{\circ}$ as substrates. Once introduced into the UHV system, they were treated by $\mathrm{Ar}$ sputter and annealing cycles in typically $10^{-6}$ mbar $\mathrm{O}_{2}$ background pressure to obtain large and homogeneous domains and the appropriate surface phase. The latter was verified by PEEM images and fitting of local X-ray-absorption spectra (XAS) and XMCD spectra to atomic multiplet calculations (for a description of the method, see Ref. 35).

For substrate preparation we adopted the following recipe: For the (110) surface, we annealed the crystals around $1000-1100 \mathrm{~K}$ in $10^{-6}$ mbar $\mathrm{O}_{2}$ for several hours. This yielded sufficiently good surface stoichiometry $\mathrm{Fe}_{3-\delta} \mathrm{O}_{4}$. $\delta=0.03$ for the (110) surface, as determined by fits of the $\mathrm{Fe}_{3} \mathrm{O}_{4} \mathrm{XMCD}$ to calculated atomic multiplet spectra (see Ref. 35 for details on the method). In Ref. 36, the appropriate stoichiometry was reported for the (110) surface prepared under similar conditions. For the (111) surface, we had to 
choose a slightly lower annealing temperature of $1000 \mathrm{~K}$ to prevent the formation of $\mathrm{FeO}$ islands. A stoichiometry analysis by the method mentioned above yielded $\delta=0.004$ for $\mathrm{Fe}_{3} \mathrm{O}_{4}(111)$; i.e., the sample was nearly stoichiometric. For the (001) surface, one expects a configuration terminated by Fe cations in octahedral coordination with oxygen, according to Ref. 37. This termination was reported to occur at a lower annealing temperature of only $850 \mathrm{~K}$. We annealed the (001)oriented sample at a slightly higher temperature of around $870 \mathrm{~K}$ and found a stoichiometry with $\delta=-0.07$ based on the analysis of the Fe XMCD signal, i.e., a stronger occupancy of the octahedral sites. This finding is consistent with the octahedral termination reported in Ref. 37, since x-ray absorption by total yield detection has a low probing depth of only $\approx 10 \AA$ at the Fe $L$ edges $^{38}$ and consequently is sensitive to the topmost atomic layers of the material.

Recnik et al. ${ }^{25}$ showed by means of transmission electron microscopy that $\mathrm{Fe}_{3} \mathrm{O}_{4} / \mathrm{NiO}$ multilayers can be grown with atomically sharp interfaces if the temperature is kept below $\approx 573 \mathrm{~K}$. In addition, Wang et al. $^{26}$ found by ultraviolet $\mathrm{X}$-ray photoemission spectroscopy (UPS/XPS) that depositing $\mathrm{NiO}$ on top of $\mathrm{Fe}_{3} \mathrm{O}_{4}$ at moderate temperatures $(<573 \mathrm{~K})$ resulted in an atomically sharp (electronic) transition from the $\mathrm{Fe}_{3} \mathrm{O}_{4}$ to the $\mathrm{NiO}$ phase. As they pointed out, however, "atomically sharp" in this sense means that a reconstruction of the interface to $\mathrm{NiFe}_{2} \mathrm{O}_{4}$ in the monolayer limit is still possible but may not be resolved by their technique. After substrate preparation, we grew $\mathrm{NiO}$ layers by oxygen-assisted molecular-beam epitaxy (MBE) in $10^{-6}$ mbar $\mathrm{O}_{2}$ background pressure at temperatures lower than $373 \mathrm{~K}$. After deposition of the $\mathrm{NiO}$ layer, the system was heated briefly above the NiO Néel temperature (525 K) and then cooled down to room temperature within several minutes in order to "freeze" the substrate domain pattern into the AF. For the (110) interface we monitored the growth of the adlayer by XAS on a NiO wedge and found sharp extrema for the XMCD signals at the $\mathrm{Fe}$ and $\mathrm{Ni}$ edges for a coverage of one monolayer. ${ }^{12}$ From this finding we conclude that the system grows with an atomically sharp interface, predominantly in a layer-by-layer fashion. Moreover, the short annealing time of $<15$ min during zero-field cooling of the $\mathrm{NiO}$ obviously did not result in an interfacial intermixing. Also for the other two orientations, we assume that we have sharp interfaces, since the uncompensated Ni magnetization is found to be very small. If, for example, Ni cations were to diffuse into the substrate, this would result in a strong Ni-XMCD signal. In fact, the signal is only $10 \%$ the size of the (110) interface. Thus we conclude that intermixing is negligible and, if at all, we have only about one reconstructed atomic layer at the interface, which presumably has a $\mathrm{NiFe}_{2} \mathrm{O}_{4}$ structure. Note that our particular deposition approach thereby avoids the formation of an extended intermediate phase region at the interface as was reported, for example, in Ref. 39.

\section{ANISOTROPIC XMLD}

In the following we will describe our approach used to extract the orientation of the spin axis in a collinear magnetic material from a set of linearly polarized x-ray-absorption spectra, i.e., exploiting the XMLD. Kuneš and Oppeneer ${ }^{29}$ were the first to predict theoretically that in the presence of a cubic crystal field, the XMLD effect becomes anisotropic. This means that the effect depends on the orientations of both spin $\mathbf{S}$ and photon polarization $\mathbf{E}$ with respect to the cubic reference frame and not, as in the isotropic case, on the relative orientation of $\mathbf{E}$ and $\mathbf{S}$ only. This result was first confirmed experimentally for $\mathrm{LaFeO}_{3}$ (Ref. 31) and shortly thereafter also for $\mathrm{Fe}_{3} \mathrm{O}_{4}$ (Ref. 32) and $\mathrm{NiFe}_{2} \mathrm{O}_{4} \cdot{ }^{33}$ Atomic multiplet calculations by van der Laan and co-workers ${ }^{31-33}$ in the above-mentioned studies confirmed the anisotropy in the XMLD and were in good agreement with the experimental data. van der Laan and co-workers showed that in cubic symmetry the XMLD spectra for arbitrary spin or polarization orientation can be calculated from only two fundamental XMLD spectra. Hereby, the angular transformation for the rotation of the magnetic field differs from the one for the polarization.

In our description we will adopt a notation which allows us to separate the material properties (magnetic and crystalline structure and symmetry) from the measurement geometry (photon incidence direction and polarization orientation). This notation is more transparent than the angular equations given in Refs. 32 and 33 for two reasons. First, it does not involve XMLD spectra, which themselves are already differences of two linearly polarized XAS spectra. We show that two fundamental XAS spectra instead of two fundamental XMLD spectra are sufficient for calculating the XAS spectra (and XMLD spectra) for arbitrary geometries. This is more convenient for experimentalists, allowing one to perform vectorial magnetometry from a set of linearly polarized XAS spectra instead a set of XMLD spectra. The compact notation we will use below is similar to formalisms applied in magneto-optics but unfortunately rarely used in $\mathrm{x}$-ray absorption spectroscopy.

In the following we will sketch the essential theoretical aspects of our technique. The linear response of a magnetic material to an oscillating electric field $\mathbf{E}(\omega)=\left|\mathbf{E}_{0}\right| e^{\imath \omega t} \boldsymbol{\epsilon}$ and photon polarization $\boldsymbol{\epsilon}$ can be described by the spin-dependent scattering form factor $\mathcal{F}(\mathbf{S}, \mathbf{Q}, \omega)$, where $\mathbf{Q}$ is the scattering vector and $\mathbf{S}$ is the vector defining the spin direction with directional cosines $[\hat{x}, \hat{y}, \hat{z}]$. The outgoing photon field is then related to the incoming photon field by $\mathbf{E}_{\text {out }}(\omega)$ $=\mathcal{F}(\mathbf{S}, \mathbf{Q}, \omega) \mathbf{E}_{\text {in }}(\omega)$. An x-ray-absorption spectrum for arbitrary polarization $\boldsymbol{\epsilon}$ and spin direction $\mathbf{S}$ can then be calculated in the limit $\mathbf{Q} \rightarrow 0$ as

$$
\mu_{\mathrm{XAS}}=-\frac{4 \pi c}{\omega} \operatorname{Im}\left\{\boldsymbol{\epsilon}^{*} \cdot \mathcal{F}(\mathbf{S}, \mathbf{Q}=0, \omega) \cdot \boldsymbol{\epsilon}\right\}
$$

For $\mathrm{NiO}$ the spin directional dependence of $\mathcal{F}$ can, within good approximation, be written as 


$$
\mathcal{F}(\mathbf{S})=\left[\begin{array}{ccc}
\mathcal{F}_{\perp}+\left(\mathcal{F}_{\|}-\mathcal{F}_{\perp}\right) \hat{x}^{2} & -\left(\mathcal{F}_{\|}-\mathcal{F}_{\perp}\right) \hat{x} \hat{y}+\imath \mathcal{F}_{\odot} \hat{z} & -\left(\mathcal{F}_{\|}-\mathcal{F}_{\perp}\right) \hat{x} \hat{z}-\imath \mathcal{F}_{\odot} \hat{y} \\
-\left(\mathcal{F}_{\|}-\mathcal{F}_{\perp}\right) \hat{x} \hat{y}-\imath \mathcal{F}_{\odot} \hat{z} & \mathcal{F}_{\perp}+\left(\mathcal{F}_{\|}-\mathcal{F}_{\perp}\right) \hat{y}^{2} & -\left(\mathcal{F}_{\|}-\mathcal{F}_{\perp}\right) \hat{y} \hat{z}+\imath \mathcal{F}_{\odot} \hat{x} \\
-\left(\mathcal{F}_{\|}-\mathcal{F}_{\perp}\right) \hat{x} \hat{z}+\imath \mathcal{F}_{\odot} \hat{y} & -\left(\mathcal{F}_{\|}-\mathcal{F}_{\perp}\right) \hat{y} \hat{z}-\imath \mathcal{F}_{\odot} \hat{x} & \mathcal{F}_{\perp}+\left(\mathcal{F}_{\|}-\mathcal{F}_{\perp}\right) \hat{z}^{2}
\end{array}\right],
$$

where $-\operatorname{Im}\left\{\mathcal{F}_{\|}\right\}$is the XAS spectrum measured for the highsymmetry case $\mathbf{S}\|\mathbf{E}\| C_{4}^{z}$ and $-\operatorname{Im}\left\{\mathcal{F}_{\perp}\right\}$ is the XAS spectrum measured for the high-symmetry case $C_{4}^{x}\|\mathbf{S} \perp \mathbf{E}\| C_{4}^{z}$. The $C_{4}$ direction is one of the fourfold high-symmetry directions given by the cubic point group, which are assumed to be in the $x, y$, and $z$ directions. $-\operatorname{Im}\left\{\mathcal{F}_{\odot}\right\}$ is the circular dichroic spectrum.

If the fundamental spectra are known, it is possible to extract the orientation of the spin $\mathbf{S}$ itself by XMCD or the axis along $\mathbf{S}$ by XMLD from a set of measured XAS spectra. For the XMLD, in general, at least three spectra have to be measured with three linear independent polarization directions to uniquely determine the spin-axis orientation. Note that in some of our experiments, only two polarizations ( $p$ and $s$ ) could be used due to the undulator design and the lack of a suitable sample rotation mimics. In that case, additional information has to be provided to produce unambiguous results, for example, by the assumption that shape anisotropy and interfacial exchange coupling confine the spins within the sample plane.

\section{EXPERIMENTAL RESULTS}

\section{A. (110) interface}

The situation at the (110) surface was already discussed in some detail elsewhere. ${ }^{12}$ For the sake of completeness and for a comprehensive discussion, however, we would like to repeat the salient features in the following. In Fig. 1 we display a typical domain pattern of the (110)-oriented $\mathrm{Fe}_{3} \mathrm{O}_{4}$ substrate recorded by means of XMCD at the $\mathrm{Fe}$ edge. Two of the easy axes of magnetite are coplanar with the (110) interface. Therefore, the resulting surface closure domains
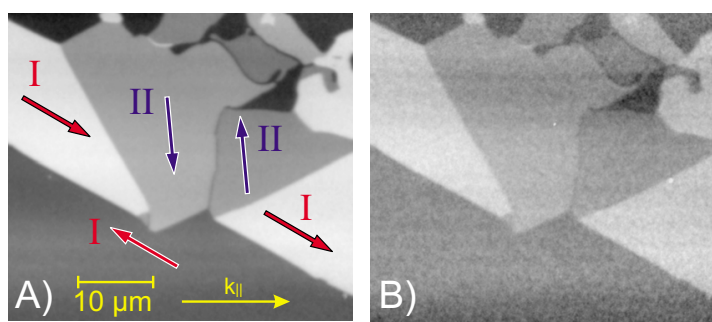

FIG. 1. (Color online) (A) XMCD contrast of the $\mathrm{Fe}_{3} \mathrm{O}_{4}$ substrate (image calculated as $\mu_{+} / \mu_{-}$at the energy of extremal XMCD amplitude). The highest and lowest gray levels correspond to the [111] easy axis (set I); the intermediate levels belong to set II ([111] axis). (B) XMCD contrast at the Ni edge, calculated analogously to that for $\mathrm{Fe}_{3} \mathrm{O}_{4}$. The contrast pattern is identical with the $\mathrm{Fe}_{3} \mathrm{O}_{4}$ XMCD. Consequently, the uncompensated magnetization at the antiferromagnet's interface is oriented parallel to the substrate magnetization. comprise two sets of $180^{\circ}$ domains, one set for each easy axis. As can be seen in Fig. 1, the stronger contrast (black and white) belongs to a magnetization vector along the [111] direction and is labeled set I. The weaker contrast belongs to magnetization directions almost perpendicular to the (horizontal) light incidence direction. It can thus be attributed to the [111] direction (set II).

In order to extract the spin-axis orientation in the $\mathrm{NiO}$ layer, we exploited the XMLD at the $\mathrm{NiO} L_{2}$ edge by calculating images of the $L_{2}$ peak ratio, calculated as the intensity of the low-energy (LE) peak divided by the intensity of the high-energy (HE) peak after subtraction of a linear background, analogous to the procedure described in Ref. 15 . The respective energies $E_{1}(\mathrm{LE})$ and $E_{2}(\mathrm{HE})$ are shown in Figs. 2(E) and 2(F). Our systems are single crystalline. Therefore the XMLD shows an anisotropic behavior in both the orientation of the light polarization $\mathbf{E}$ and the spin $\mathbf{S}$ with respect to the cubic lattice frame, according to Eqs. (1) and (2).

At this point it should be pointed out clearly that for the vectorial magnetometry, i.e., the extraction of the spin-axis orientation by measuring the XMLD contrast in different experimental geometries, it is the angular dependence of the XMLD which is essential for the interpretation of the data. Considering the experimental geometry (crystal orientation, light incidence, and polarization direction), one obtains reliable predictions on the contrast between different magnetic domains in the sample, which can be easily compared to PEEM measurements. The absolute magnitude of the XMLD, which depends on temperature and experimental resolution, is a secondary aspect in this process, i.e., does not affect the validity of our results in terms of the spin-axis orientations derived from experiment.

However, a quantitative comparison of our experimental contrast to other those of experiments is still recommended, so we used the data on $\mathrm{NiO} / \mathrm{MgO}(001)$ by Alders et al. ${ }^{15}$ as a reference to extract the fundamental XAS spectra of NiO. Please note at this point that different experimental conditions in the experiment of Alders et al. and ours will definitely lead to different magnitudes of the XMLD contrast. For example, the contrast decreases with increasing temperature as well as with decreasing experimental resolution. Since both experiments were done at room temperature, the influence of thermal effects should be small. From our NiO spectra in Figs. 2(E) and 2(F), we deduce a slightly decreased experimental resolution of $0.6 \mathrm{eV}$ as compared to that of Alders et al. $(\approx 0.45 \mathrm{eV})$. This implies that the measured magnitude of our XMLD contrast will always be somewhat lower than the predictions made with the help of the values of Alders et al.

To test the validity of the interpretation of the XMLD contrast given in Ref. 15, we used two different scenarios in 


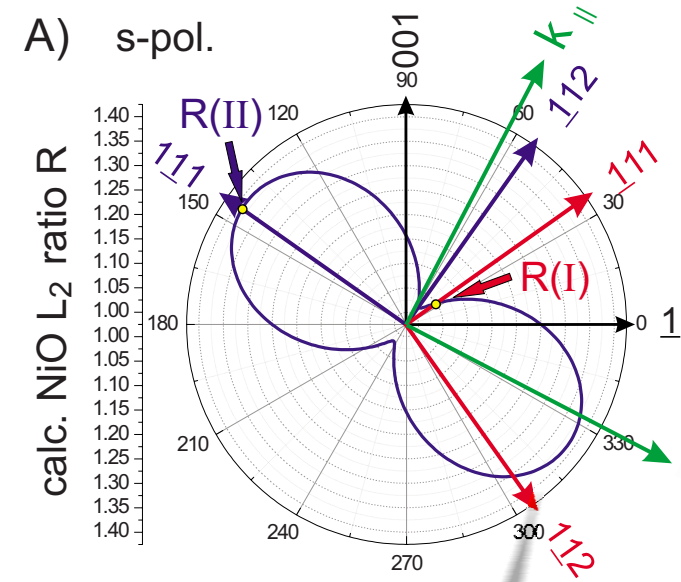

C)

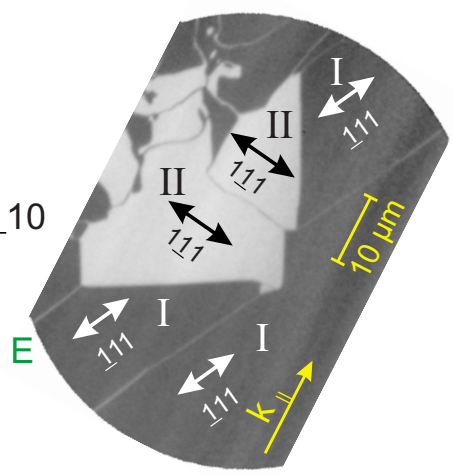

D)

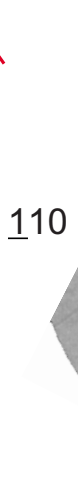

E)

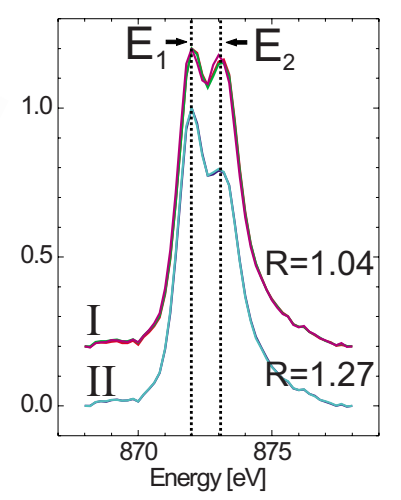

F)

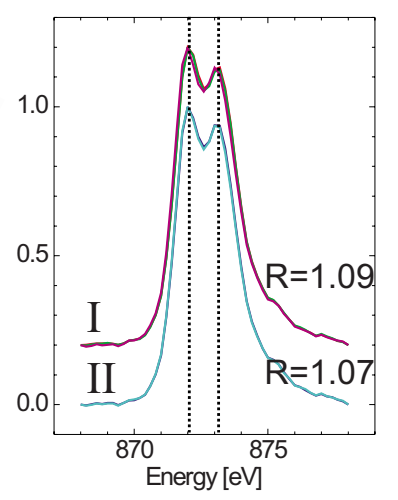

FIG. 2. (Color online) 35 monolayers (MLs) $(51 \AA)$ of $\mathrm{NiO}$ on $\mathrm{Fe}_{3} \mathrm{O}_{4^{-}}(110)$. The polar plots show the calculated $L_{2}$ ratio ( curve) for every possible direction of the spin within the (110) plane, with the polarization fixed to $s$ geometry (A) or $p$ geometry (B). In the polar plots, the crystallographic directions for collinear and spin-flop coupling are shown, as well as the surface light incidence projection $\mathbf{k}_{\|}$and polarization E. The expected PEEM contrast for a given spin orientation in the sample plane is defined by the intersection of this direction with the $L_{2}$-ratio curve. In (C) and (D), the experimental PEEM contrast is shown for the two geometries. Contrast in $p$ geometry has been enhanced for better visibility. Panels (E) and (F) show the respective microspectra within the different domain sets I and II. For collinear coupling we assign the axis [111] to set I and [1 11$]$ to set II. Conversely for spin-flop coupling, the assignment is [1 12$]$ for set I and [112] for set II. Only the collinear case matches the theoretically predicted contrast, with set II being brighter in $s$ geometry and slightly darker in $p$ geometry [points $\mathrm{R}(\mathrm{I})$ and $\mathrm{R}(\mathrm{II})$ in (A) and (B)]. Spin-flop coupling would produce a reversed contrast and can thus be excluded.

extracting the fundamental spectra: (i) a $\mathrm{NiO} \mathrm{AF}$ domain state with only out-of-plane $[ \pm 1 \pm 1 \pm 2]$ easy axes-as originally assumed by Alders et al. ${ }^{15}$-and (ii) a spin axis along the sample normal [001] ( $z$-aligned case). Both scenarios will result in an angular dependence of the XMLD, which is rotationally symmetric to the sample normal-in good agreement with the experiment of Alders et al. However, the size of the XMLD contrast will be different. In our case, assumption (i) produces deviations (up to 30\%) between the calculated ratio and our experimental $L_{2}$ ratio, while assumption (ii) leads to values which differ only by at most $10 \%$ from our data. The predicted PEEM contrast between different AF domains is larger than our experimental contrast for both cases, indicating that indeed our lower experimental resolution is the cause of the deviations. It is, however, interesting to note that the $z$-aligned case, (ii), can also better account for the maximum XMLD contrast found by Ohldag ${ }^{40}$ in a single domain of a $\mathrm{NiO}$ crystal, who also had a resolution lower than that in the experiments of Alders et al..$^{15}$
In the following, we will choose scenario (ii), the $z$-aligned case, mainly because the data fit better to our experiment. Note that we do not claim that the interpretation of Alders et al. ${ }^{15}$ is wrong, since we do not have enough significance to exclude scenario (i). Certainly, more experimental work has to be done to quantify the effect of anisotropic XMLD in thin films of $\mathrm{NiO}$ as compared to single crystals.

Finally, using the derived data in Eqs. (1) and (2), we are able to calculate the $\mathrm{NiO} L_{2}$ ratio for all possible orientations of the spin $\mathbf{S}$. Calculations were performed for both polarization configurations available in experiment, namely, $p$ contrast ( $\mathbf{E}$ is mainly out of plane) and $s$ contrast ( $\mathbf{E}$ is in plane).

Our further evaluation yielded that the spin axis in $\mathrm{NiO}$ is in plane. For the sake of clarity, we therefore restrict ourselves to plotting the anisotropy of the XMLD within the sample plane. The theoretical in-plane contrast is shown in Figs. 2(A) and 2(B) together with the corresponding PEEM images in (C) and (D) and $\mathrm{NiO} L_{2}$ microspectra in (E) and (F). Furthermore, a numerical comparison between calcu- 
TABLE I. Experimental and calculated values for the $\mathrm{NiO} L_{2}$ ratio in the domain sets I and II. Two possible coupling geometries are compared, namely, collinear and spin-flop couplings. It is directly apparent that spin-flop coupling produces a sign reversal of the theoretically computed contrast (domain set II-domain set I) as compared to experiment (typeset in italic). In contrast to this, the collinear case results in good accordance between theory and experiment.

\begin{tabular}{cccccrc}
\hline \hline & & \multicolumn{3}{c}{ Collinear coupling } \\
Polarization & \multicolumn{2}{c}{ Set I } & \multicolumn{2}{c}{ Set II } & \multicolumn{2}{c}{ Difference (II-I) } \\
& Expt. & Theor. & Expt. & Theor. & Expt. & Theor. \\
\hline$s$ & 1.04 & 1.05 & 1.27 & 1.39 & 0.23 & 0.34 \\
$p$ & 1.09 & 0.99 & 1.07 & 0.96 & -0.02 & -0.03 \\
& & & & & & \\
$s$ & 1.04 & 1.35 & 1.27 & 1.02 & 0.23 & -0.33 \\
$p$ & 1.09 & 1.09 & 1.07 & 1.11 & -0.02 & 0.02 \\
\hline \hline
\end{tabular}

lated and measured contrast for both collinear and spin-flop couplings is given in Table I.

The coupling geometry can be extracted by comparing the domain contrasts in sets I and II for both available polarizations $p$ and $s$. This can be done by calculating the difference between the $L_{2}$ ratios (set II-set I) as shown in Table I. Comparing experimental and theoretical values, we can definitely exclude perpendicular coupling (set I: in-plane [112]; set II: [112] direction), since it would imply a reversal of the magnetic contrast as compared to the experiment (see numbers typeset in italic in Table I). Choosing, however, the [111] direction for set I and the [111] direction for set II, the contrast is reproduced correctly. For example, in $s$ contrast, theory predicts set II to be considerably brighter than set I, while for $p$ contrast, set II should be only marginally darker than set I. This trend is clearly reflected in the PEEM images in Figs. 2(C) and 2(D). Thus, we can safely conclude that at the (110) interface, a collinear coupling between a ferrimagnet and an antiferromagnet is realized.

\section{B. (001) interface}

The magnetic structure in the case of $\mathrm{Fe}_{3} \mathrm{O}_{4}(001) / \mathrm{NiO}$ was determined from XMLD measurements at the BESSY UE-56/1 beamline as well as the SIM beamline of the Swiss Light Source (Paul Scherrer Institut, Switzerland). The latter allows one to rotate the linear polarization direction around the beam axis, which yields more conclusive results for the spin-axis orientation by angular scans of the polarization. For a quick overview, we show both PEEM images and microspectra of the $\mathrm{Fe}_{3} \mathrm{O}_{4}(001) / \mathrm{NiO}$ system in Fig. 3, taken during sample precharacterization at BESSY. Image (A) shows the $\mathrm{Fe}_{3} \mathrm{O}_{4}$-XMCD contrast, which was calculated as a ratio of two images, $\mu(\sigma+) / \mu(\sigma-)$, at the energy of extremal $\mathrm{XMCD} E_{3}$. Since the easy axes of magnetite do not coincide with the surface plane, a complicated quasidomain pattern results with "fir-tree"-like structures in order to compensate the out-of-plane magnetization component of the volume domains underneath. The magnetization in the lamellae oscillates around the projections of the bulk easy axes on the crystal surface, which are the $\langle 110\rangle$ directions.
The NiO-XMLD image in Fig. 3(B) was calculated by the standard rule as the ratio of the LE/HE peak intensities of the $L_{2}$ edge, $\mu\left(E_{1}\right) / \mu\left(E_{2}\right)$. The $\mathrm{Fe}_{3} \mathrm{O}_{4}$-XMLD image which is not shown in the figure shows a contrast similar to that in Fig. 3(B). It represents a division of the intensities at two energies of extremal $X M C D$ in the $L_{3}$ edge of magnetite $\left[\mu\left(E_{3}\right) / \mu\left(E_{4}\right)\right]$. As one can see in Fig. 3(D), the XMLD signal calculated from the $s$-polarized microspectra corresponds roughly to $\mu(\mathbf{S} \perp \mathbf{E})-\mu(\mathbf{S} \| \mathbf{E})$. The XMLD is quite large at the energies $E_{3}$ and $E_{4}$, producing a good signal-to-noise ratio. The spectral shape of the XMLD corresponds well to the findings in Ref. 32.
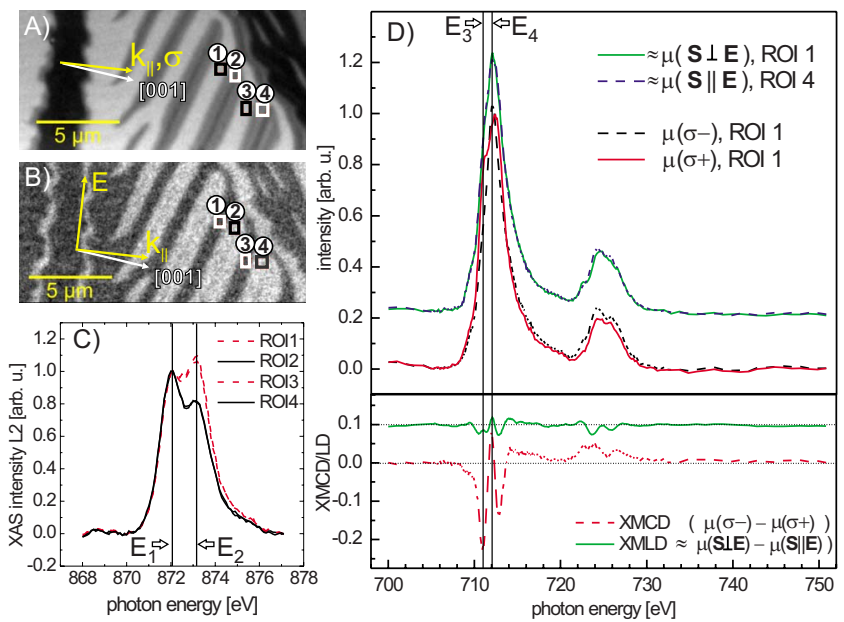

FIG. 3. (Color online) Local microspectra taken for the $\mathrm{Fe}_{3} \mathrm{O}_{4}(001) / \mathrm{NiO}$ interface. (A) $\mathrm{Fe}_{3} \mathrm{O}_{4}$-XMCD PEEM image calculated as $\mu_{+} / \mu_{-}$at the energy $E_{3}$ of extremal XMCD amplitude. (B) $\mathrm{NiO}-\mathrm{XMLD}$ image with $s$ polarization, representing the $L_{2}$ ratio calculated as $\mu\left(E_{1}\right) / \mu\left(E_{2}\right)$. (C) $\mathrm{NiO} L_{2}$ microspectra from ROIs 1-4. (D) $\mathrm{Fe}_{3} \mathrm{O}_{4}$ microspectra. The XMLD nearly corresponds to the situation $\mu(\mathbf{S} \perp \mathbf{E})-\mu(\mathbf{S} \| \mathbf{E})$. Note that the PEEM images of the $\mathrm{Fe}_{3} \mathrm{O}_{4}$-XMLD contrast (not shown here) have been calculated as $\mu\left(E_{3}\right) / \mu\left(E_{4}\right)$. Finally, the XMCD spectra were calculated from ROI 1 , where the substrate spin points nearly along the light incidence projection $\mathbf{k}_{\|}$(maximum XMCD contrast). Note that the energies $E_{1-4}$ for PEEM contrast generation have been marked in all spectra. 

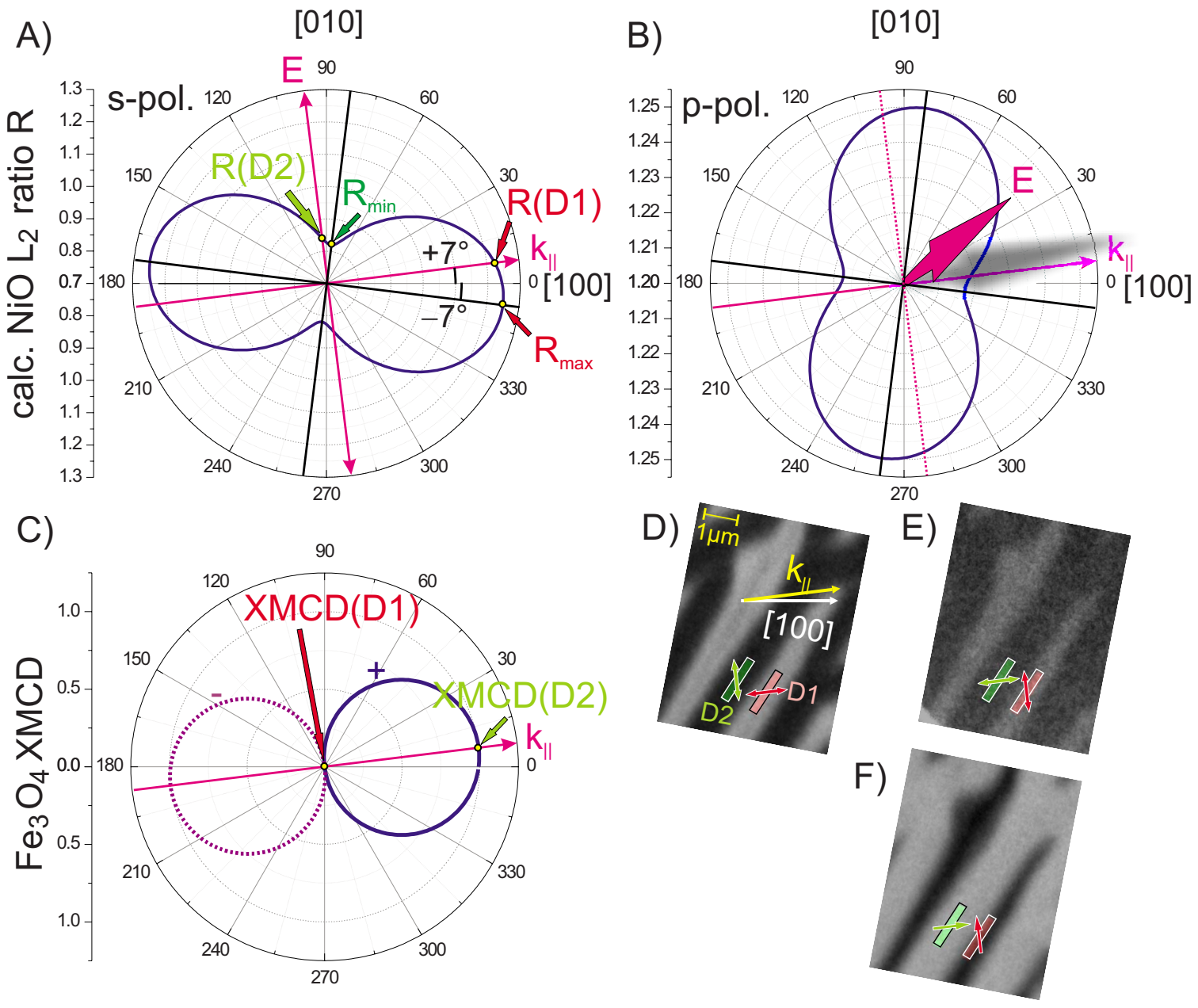

FIG. 4. (Color online) Calculated angular dependence of the $\mathrm{NiO} L_{2}$ ratio when varying the spin direction within the (001) plane, calculated for $s$ polarization (A) and $p$ polarization (B). Panel (C) shows the angular dependence of the XMCD for comparison. The PEEM images show the $s$-polarized NiO-XMLD contrast (D), $\mathrm{Fe}_{3} \mathrm{O}_{4}$-XMLD contrast (E), as well as the $\mathrm{Fe}_{3} \mathrm{O}_{4}$-XMCD contrast (F). ROIs D1 and D2 for the angular scans, as shown in the images, are chosen as follows: In D1, the magnetization in the substrate is perpendicular to the in-plane projection of the light propagation direction $\left(k_{\|}\right)$, while in D2, it is parallel (see the red and green arrows). If the NiO layer couples spin flop, its spin-axis directions then point along the axes represented by the double arrows in (D). Region D1 then produces almost maximum XMLD contrast, while region D2 shows nearly minimal signal. Comparing the XMCD angular dependence in (C) with image (F), one finds intermediate contrast (no XMCD signal) for D1 and maximum contrast/XMCD signal for D2. Note that for collinear coupling, the labels D1 and D2 in the NiO XMLD [(A) and (D)] would have to be interchanged. The anisotropic XMLD reveals itself through a phase shift between the XMLD and XMCD angular dependencies. Since $\mathbf{E}$ in $s$ geometry forms an angle of $7^{\circ}$ with the [010] axis, the maximum of the XMLD anisotropy deviates by $-7^{\circ}$ from that direction.

For the angular scans, the polarization orientation was varied gradually from $s$ to $p$ polarization. For a start, one has to choose two different regions on the sample, where the spin-axis orientations are extremal with respect to the rotation plane, i.e., parallel or perpendicular. In this way a maximum sensitivity of the measurement can be achieved. Assuming that the demagnetizing field confines the substrate magnetization in plane, we selected one region of nearly vanishing XMCD signal (D1), i.e., with the $\mathrm{Fe}_{3} \mathrm{O}_{4}$ spin direction supposedly perpendicular to the light incidence projection on the sample surface $\left(\mathbf{S} \perp \mathbf{k}_{\|}\right)$, and another one with $\mathbf{S} \| \mathbf{k}_{\|}$. The expected XMCD PEEM contrast is then intermediate for D1 and maximum (minimum) for D2 [compare Fig. $4(C)$, which agrees well with the finding in image $(F)$ of Fig. 4.
In order to see why the NiO-XMLD contrast is nearly extremal for the two regions, we have to look at Fig. 4, where the theoretical curves for the $\mathrm{NiO} L_{2}$ ratio are plotted for a hypothetical full in-plane rotation of the $\mathrm{NiO}$ spin axis with an orientation perpendicular to the $\mathrm{Fe}_{3} \mathrm{O}_{4}$ net moment. ${ }^{41}$ A simple comparison of the NiO-XMLD image in $s$ polarization Fig. 4(D) to the polar plot in Fig. 4(A) already yields a coarse estimate of the coupling angle: The scenario of perpendicular coupling is more realistic because the $\mathrm{NiO}$ XMLD signal then produces almost maximum contrast for D1 and minimum for D2 - in accordance with the PEEM data-whereas collinear coupling would yield the opposite behavior.

Note that the XMLD contrast variation also exhibits a phase shift with respect to the XMCD dependency, as can be 


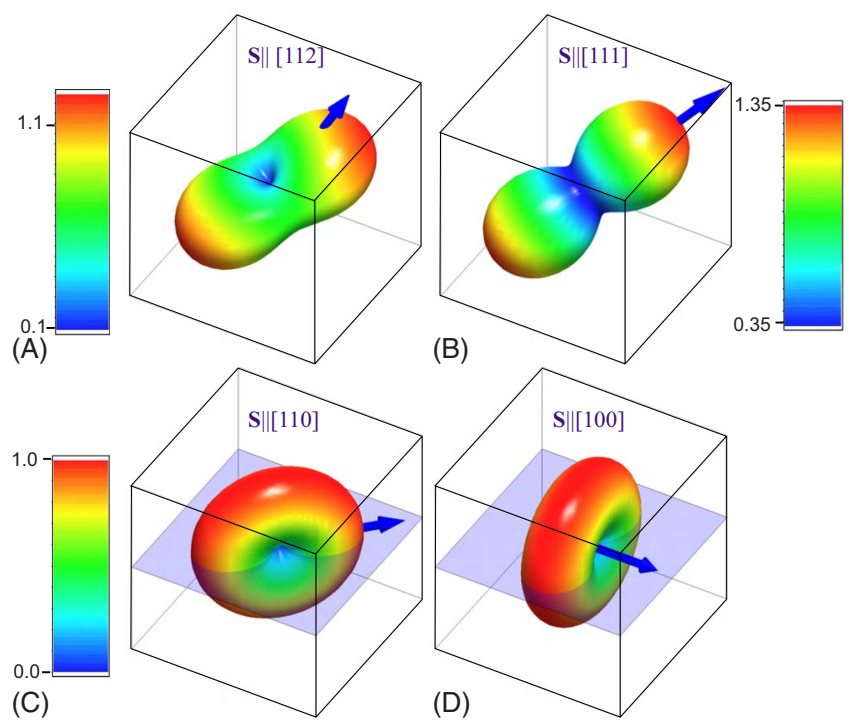

FIG. 5. (Color online) Demonstration of the XMLD manifold, calculated from Eqs. (1) and (2): Each surface represents the set of all linearly polarized XAS intensities at a given photon energy $\hbar \omega$ for a fixed spin-quantization axis (blue arrow). For simplicity, we fixed the values of $\mathcal{F}_{\|}(\omega)$ and $\mathcal{F}_{\perp}(\omega)$ to 0 and 1 , respectively. Shown are the four spin orientations $\mathbf{S} \|[112]$ (A), $\mathbf{S} \|[111]$ (B), $\mathbf{S} \|[110]$ (C), and $\mathbf{S} \|[100]$ (D). Note that when rotating the spin from $\mathbf{S} \|[001]$ to $\mathbf{S} \|[110]$, i.e., rotating by $+45^{\circ}$, the XMLD manifold rotates by $-45^{\circ}$, i.e., by $90^{\circ}$ with respect to the spin axis. Consequently, when the XMLD contrast is calculated within the (001) plane as $\mu(\mathbf{E} \| \mathbf{S})-\mu(\mathbf{E} \perp \mathbf{S})$, it inverts upon changing the spin axis from $\mathbf{S} \|[001]$ to $\mathbf{S} \|[110]$. This simple example shows that erroneous conclusions about the spin-axis orientation can result if the anisotropic XMLD is not considered. For more details, see Ref. 42.

seen in Fig. 4(A), for example. Whereas the XMCD signal is maximum along $k_{\|}$, which forms an angle of $+7^{\circ}$ with the [001] direction, the maximum of the XMLD signal is shifted away by $-7^{\circ}$ from this direction. This is a consequence of the anisotropic nature of the XMLD. The angular dependence described in Eqs. (1) and (2) predicts the following: For a polarization oriented within a $C_{4}$ plane of the cubic solid, one can construct the polarization-dependent manifold of the XMLD contrast, i.e., the set of all spin-dependent XAS spectra for a fixed polarization axis, sweeping the spin over the full solid angle. This manifold possesses rotational symmetry to an axis within the $C_{4}$ plane, which coincides with the polarization direction for a $C_{4}$ axis, and will otherwise rotate in antisense to any polarization rotation within the $C_{4}$ plane. Rotating the polarization away from this axis by an angle $\phi$ within the (001) plane results in rotation of the XMLD manifold by $-\phi$. Hence the XMLD signal will exhibit a phase shift of $2 \phi$, as can be seen in the plot in Fig. 4(A). It is also very important to note that using Eq. (2) in Eq. (1), the quantities $\mathbf{S}$ and $\mathbf{E}$ commute, i.e., the shape of the manifold for a particular geometry will stay the same when exchanging $\mathbf{E}$ and $\mathbf{S}$, keeping $\mathbf{S}$ fixed and sweeping $\mathbf{E}$. An important consequence is that the contrast levels of the XMLD for the two cases $\mathbf{E} \| \mathbf{S}$ and $\mathbf{E} \perp \mathbf{S}$ are interchanged when going from a $\mathbf{S} \|[110]$ to a $\mathbf{S} \|[100]$ configuration, since
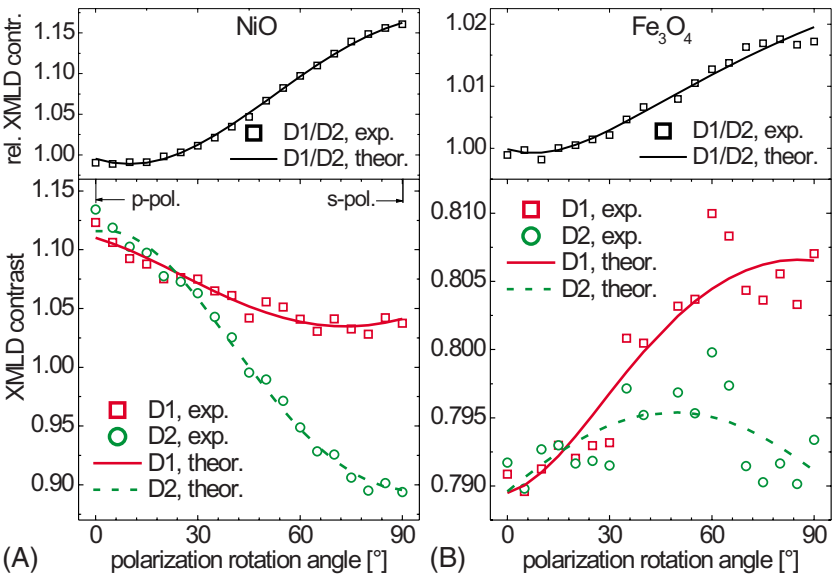

FIG. 6. (Color online) XMLD contrast for angular polarization scans from a $43 \AA$ (21 MLs) NiO layer on $\mathrm{Fe}_{3} \mathrm{O}_{4}(001)$. The corresponding regions D1 and D2 are shown in Figs. 4(D)-4(F). The polarization was varied from $p\left(0^{\circ}\right)$ to $s\left(90^{\circ}\right)$, taking PEEM parameter images at each angle. (A) $\mathrm{NiO} L_{2} \mathrm{XMLD}$, representing the $L_{2}$ ratio $\mu(E 1) / \mu(E 2)$. (B) $\mathrm{Fe} L_{3} \mathrm{XMLD}\left(\mathrm{Fe}_{3} \mathrm{O}_{4}\right)$, representing the ratio $\mu\left(E_{3}\right) / \mu\left(E_{4}\right)$. From the fits (solid and dashed lines), the tendency of the system for spin-flop coupling can be derived (see text).

the relative orientation of the XMLD manifold with respect to the spin axis will be rotated by exactly $90^{\circ}$ [compare Figs. $5(\mathrm{C})$ and 5(D)]. This particular phenomenon is the reason why some of the earlier findings in (001)-oriented systems have to be reconsidered, since they were interpreted on the basis of the isotropic XMLD theory (for example, Refs. 8, 9, 11, 34, and 43). Under these circumstances an error in the spin-axis orientation of $90^{\circ}$ is easily possible, and in coupled systems spin-flop coupling can easily be mistaken for collinear coupling, as was, for example, verified by Arenholz et $a l .{ }^{33}$ in the case of Ref. 9 . In conclusion, it is mandatory to take into account the anisotropic characteristics of the XMLD effect for a reliable vectorial magnetometry in crystalline systems.

After definition of the regions of interest (ROIs), angular scans of the light polarization from $p$ to $s$ geometry were performed. The corresponding contrast variation for $\mathrm{Fe}_{3} \mathrm{O}_{4}$ and $\mathrm{NiO}$ is shown in Fig. 6. Comparing the $\mathrm{Fe}_{3} \mathrm{O}_{4}$ - and $\mathrm{NiO}$ XMLD signals, an inverse behavior of the angular variation in the XMLD contrast between D1 and D2 is apparent. While D1 shows a weak angular variation for $\mathrm{NiO}$, it changes strongly for $\mathrm{Fe}_{3} \mathrm{O}_{4}$. For D2, the behavior is opposite. From this finding, one can already conclude on a noncollinear orientation of the spin axes in the ferro- and antiferromagnet.

A more complete picture is finally obtained from the explicit comparison of the experimental results to a theoretical treatment of the anisotropic XMLD. For this purpose, the theoretical MLD contrast variation produced by Eqs. (1) and (2) was fitted to the experimental curves of both $\mathrm{NiO}$ and $\mathrm{Fe}_{3} \mathrm{O}_{4}$, taking the spin directions of D1 and D2 extracted from the XMCD analysis as starting values and assuming either an in-plane spin-flop coupling or-for a consistency check - a collinear arrangement. While for the collinear case the fit yielded a negative scaling parameter for the NiO MLD (which would contradict the atomic multiplet calculations) 
together with a poor convergence, the parameter is positive for the spin-flop case and the fit converges well.

The ratio curves D1/D2 show the good fit quality, since all correlated noise contained in the separate measurements of D1 and D2 is removed. ${ }^{44}$ Together with the coupling angles derived from the fits $\left(\alpha_{\mathrm{D} 1}=58 \pm 10^{\circ}\right.$ and $\left.\alpha_{\mathrm{D} 2}=103 \pm 10^{\circ}\right)$, one can directly conclude that collinear coupling can be ruled out and instead a spin-flop state is realized in the antiferromagnet. Furthermore, the results show that the $\mathrm{NiO}$ spins are not oriented out of plane, as could be expected from the deformation of the $\mathrm{NiO}$ layer due to a tensile inplane strain, ${ }^{14,16}$ but are lying almost in plane (angle to the surface $<9^{\circ}$ ). This is very likely caused by the interfacial exchange coupling to the substrate magnetization, which is forced to be in plane due to the shape anisotropy. Our results at the (001) interface are in accordance with the findings for $\mathrm{Fe}_{3} \mathrm{O}_{4}(001) / \mathrm{NiO}$ superlattices, for which spin-flop coupling has been reported as well. ${ }^{45,46}$

Finally, it is interesting to note that the angular transformation in Eq. (2), which has been gained from atomic multiplet calculations of $\mathrm{NiO}\left(\mathrm{Ni} d^{8} \mathrm{O}_{h}\right)$, produces such a good fit quality for $\mathrm{Fe}_{3} \mathrm{O}_{4}$ as well. This circumstance is nontrivial, since magnetite is a mixed-valence compound with spinel structure, i.e., two different types of site symmetry, namely, octahedral and tetrahedral. Furthermore, it contains Fe cations with orbital degrees of freedom (high-spin $d^{6}$ ), in contrast to $\mathrm{NiO}$ (high-spin $d^{8}$ ). The finding that the same angular transformations are applicable for both $\mathrm{NiO}$ and $\mathrm{Fe}_{3} \mathrm{O}_{4}$ is, however, in good agreement with the results in Refs. 32 and 33. We point out that the angular transformation in Eq. (2) is not generally transferrable to arbitrary cubic compounds.

\section{C. (111) interface}

Finally we turn to the (111) orientation. Measurements for this interface have been performed at the BESSY beamline UE 56/1 SGM, which provides linearly and circularly polarized light but no free rotation of the light polarization. Fortunately, the intrinsic domain pattern of the $\mathrm{Fe}_{3} \mathrm{O}_{4}(111)$ surface offered an interesting and convenient way to work around this limitation. The quasidomain fir-tree-type pattern at the (111) surface consists of a network of lamellar structures. In each of these lamellae, a quasicontinuous rotation of the magnetization vector in the film plane takes place. ${ }^{47}$ Due to the presence of all possible in-plane magnetization directions in one PEEM image, the lateral distribution of contrast levels can be analyzed in the sense of a quasirotation experiment, and a rotation of the sample or polarization vector of the light becomes obsolete. XMCD line profiles perpendicular to the lamellae main axis record a full in-plane turn of the substrate magnetization, and the corresponding XMLD profiles show the related behavior of the spin axis in $\mathrm{NiO}$. What remains to be done is a comparison of the calculated NiOXMLD profile to the experimental profile. This analysis has been performed in Fig. 7. On the left-hand side the profiles for NiO-XMLD contrast ( $p$ and $s$ geometries) as well as $\mathrm{Fe}_{3} \mathrm{O}_{4}$-XMCD contrast are shown. From the comparison of experiment and theory it is obvious that the minima of the $s$-polarization XMLD signal coincide in most cases with the

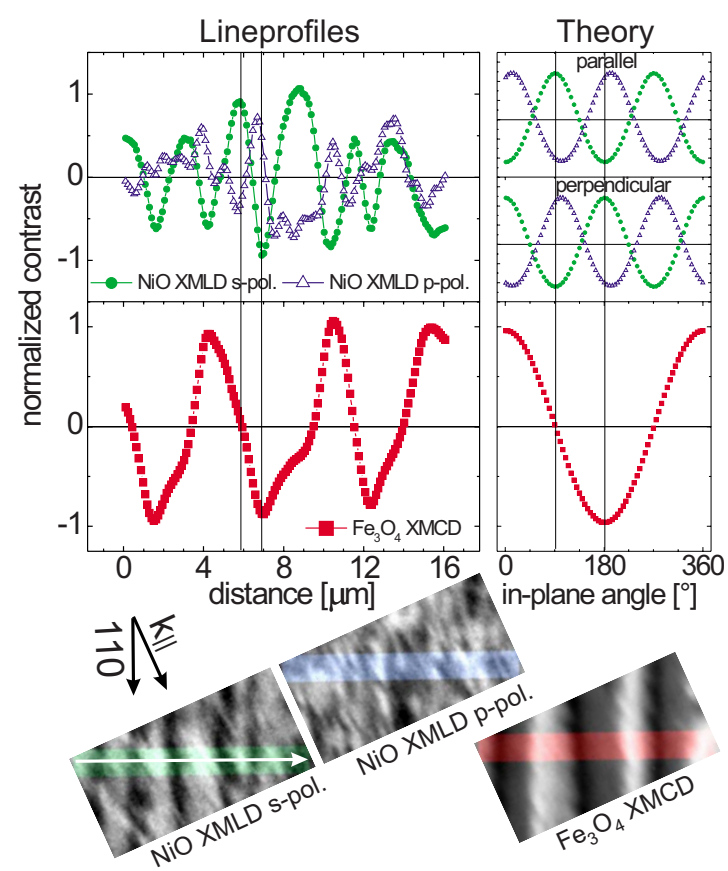

FIG. 7. (Color online) Interfacial coupling in $\mathrm{Fe}_{3} \mathrm{O}_{4}(111) / \mathrm{NiO}[37 \AA]$. Upper left: Experimental line profiles. Upper right: Theoretical profiles calculated for a full in-plane rotation of $\mathbf{S}$ for both collinear and perpendicular coupling. Lower panel: Corresponding PEEM division images, calculated by the standard procedure. Laue measurements confirm that the stripe pattern is parallel to a $\langle 110\rangle$ direction.

extrema of the XMCD signal, whereas the maxima tend to coincide with the zero-crossings of the XMCD. This situation corresponds to the simulation of a collinear coupling situation shown in the right-hand panels. We would like to point out that a perpendicular coupling would just result in the opposite behavior. Note that the $p$-polarized contrast has a slight phase shift with respect to the $s$-polarized data-as stated earlier-because $\mathbf{E}$ does not point along any highsymmetry directions. The particular feature of the $p$-polarization contrast of having maxima near the minima of the $s$-polarization contrast can also be observed experimentally. However, the data quality is inferior to the one obtained in the measurement for $s$-polarization contrast. To conclude, the (111) interface in the $\mathrm{Fe}_{3} \mathrm{O}_{4} / \mathrm{NiO}$ system assumes a collinear coupling, just as the (110) interface does, in contrast to the (001) interface.

\section{Orientation dependence of the uncompensated magnetization}

The above results clearly demonstrate the interface orientation to be a critical parameter in determining the coupling scheme. It is therefore reasonable to expect that the amount of uncompensated magnetization at the interface will depend on this orientation, too. To corroborate this hypothesis, we performed a more detailed analysis of the NiO-XMCD signal. For this purpose, we searched the extremal XMCD amplitude for both $\mathrm{Fe}_{3} \mathrm{O}_{4}$ and $\mathrm{NiO}$, which is found to be negative at the $L_{3}$ edges (compare Ref. 12), and we calculated 


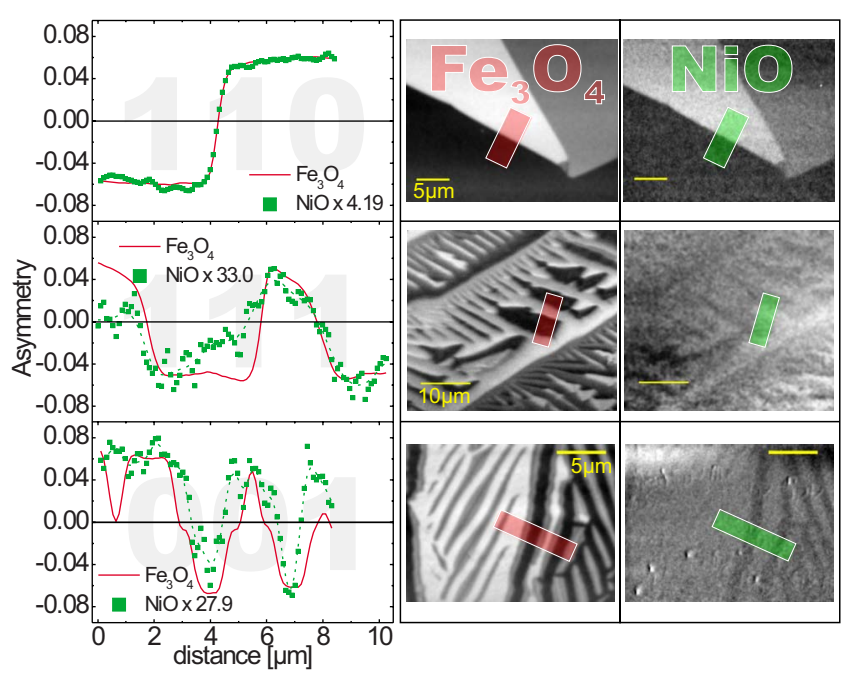

FIG. 8. (Color online) Comparison of the amount of uncompensated magnetization at the interface for the three different orientations (110), (111), and (001). For comparison, the NiO XMCD has always been scaled to match the size of the $\mathrm{Fe}_{3} \mathrm{O}_{4} \mathrm{XMCD}$, which was assumed not to depend on the interface orientation.

asymmetry images $\left(\mu_{+}-\mu_{-}\right) /\left(\mu_{+}+\mu_{-}\right)$. Since the magnetic moment of $\mathrm{Fe}_{3} \mathrm{O}_{4}$ is assumed to vary little with interface orientation, the respective XMCD signal is used as a stable reference, to which the NiO-XMCD signal is set in relation. In this way, we obtain scaling factors which vary with the interface orientation. The results of this evaluation are shown in Fig. 8.

Since the (110) interface exhibits the largest uncompensated magnetization in the AF, we used it as a reference in the comparison of the three interfaces. For reasons of simplicity we assume that the Ni spins at the (110) interface are aligned fully parallel to the substrate magnetization. The second simplifying assumption is that only one interfacial monolayer will contribute to the NiO-XMCD. Although in reality the proximity zone may spread over two to three monolayers, the integrated signal will roughly be equivalent to one monolayer, since the effects decay quickly with distance from the interface. Thus, our simplification is justified. Last but not least, the signals have to be corrected for the slightly different film thicknesses of the various samples by taking into account the electron escape depth $\lambda_{e}$ and the normal x-ray-absorption length $\lambda_{x}^{\prime}=\lambda_{x} \sin (\theta)$, where $\theta$ is the incidence angle to the plane $\left(16^{\circ}\right)$. The NiO-XMCD signal from a buried region ranging from the depth $\tau$ to $\tau-d_{l}$ is proportional to

$$
\begin{gathered}
I_{\mathrm{XMCD}, \mathrm{NiO}} \propto \frac{\int_{0}^{d_{l}} e^{-\mu_{\mathrm{eff}}(\tau-z)} d z}{\int_{0}^{\tau-d_{l}} e^{-\mu_{\mathrm{eff}}} d z}=-\frac{e^{-\mu_{\mathrm{eff}} \tau}\left(1-e^{+\mu_{\mathrm{eff}} d_{l}}\right)}{1-e^{-\mu_{\mathrm{eff}}\left(\tau-d_{l}\right)}}, \\
\mu_{\mathrm{eff}}=\frac{\lambda_{e}+\lambda_{x}^{\prime}}{\lambda_{e} \lambda_{x}^{\prime}},
\end{gathered}
$$

while the escaping electrons from $\mathrm{Fe}_{3} \mathrm{O}_{4}$ have to travel through the $\mathrm{NiO}$ layer of thickness $\tau$ and are reduced in number by a factor of $\exp \left\{-\mu_{\mathrm{eff}} \tau\right\}$. Thus, relating the $\mathrm{NiO}$ contrast to the $\mathrm{Fe}_{3} \mathrm{O}_{4}$ contrast will result in an absorption dependence

$$
\gamma=\frac{I_{\mathrm{XMCD}, \mathrm{Fe}_{3} \mathrm{O}_{4}}}{I_{\mathrm{XMCD}, \mathrm{NiO}}} \propto-\frac{\left(1-e^{+\mu_{\mathrm{eff}} d_{l}}\right)}{1-e^{-\mu_{\mathrm{eff}}\left(\tau-d_{l}\right)}} .
$$

Correcting with $1 / \gamma$ from Eq. (4), the scaling values for the (001) and (111) interfaces can now be related to those obtained for the (110) orientation, yielding the amount of uncompensated magnetization in percent. ${ }^{48}$ For the (111) interface, we obtain $13.8 \pm 7 \%$, while for the (001) interface the value is $9.8 \pm 3 \%$. Since spin-flop coupling occurs at the (001) interface, we can also assign a canting angle of the sublattice spins toward the substrate magnetization direction, which is only about $5.6 \pm 2^{\circ}-$ a typical value for spin-flop systems as found by Koon. ${ }^{22}$ Of course, this is an upper limit, since it is always possible that magnetic defects and not canting alone are responsible for the uncompensated magnetization. Those can be located at step edges or inside antiphase boundaries in the antiferromagnet. They usually have a lower anisotropy and can align more easily with the substrate magnetization.

\section{DISCUSSION OF THE RESULTS}

\section{A. Influence of the interface structure on the coupling type}

Applying Koon's theory in a naive way, one may postulate spin-flop or collinear coupling if the AF surface termination is compensated or uncompensated, respectively. Applied to our case, we then would expect spin-flop coupling for a ferromagnet in contact with the compensated (110) and (001) NiO surfaces. Comparing to our experiments, it becomes clear that for the (110) interface, the experimental situation is different (collinear coupling). We have to keep in mind, however, that the interface has two sides, i.e., a $\mathrm{NiO}$ and an $\mathrm{Fe}_{3} \mathrm{O}_{4}$ side. It is thus clear that the naive approach is too simple and one has to consider the full crystalline and magnetic structure of the transition $\mathrm{Fe}_{3} \mathrm{O}_{4}$ (ferrimagnetic, spinel) $\rightarrow \mathrm{NiO}$ (two sublattice, rocksalt). In all three crystalline orientations investigated, one can find a configuration where the magnetic unit cells of $\mathrm{NiO}$ and $\mathrm{Fe}_{3} \mathrm{O}_{4}$ match. This implies that the two magnetic sublattices (spin up and spin down) of $\mathrm{NiO}$ will experience different magnetic environments at the interface. Consequently, in a more realistic picture the interface cannot be compensated anymore and a tendency for collinear coupling should result-an argument which was also given by van der Zaag et al. ${ }^{23}$ The extreme case is the (111) interface, where locally only one type of AF sublattice meets the interface (locally uncompensated). In principle, partial compensation is realized for all interface orientations investigated in this study, rendering spin-flop coupling unlikely. Experimentally, however, we find perpendicular coupling for the (001) interface, contradicting the structural argument given above. Therefore, we have to consider additional mechanisms which favor a perpendicular over a collinear spin arrangement, overcoming the influence of exchange interactions and the particular interface structure. Such mechanisms may be of magnetoelastic origin, as 
will be discussed in the following paragraphs.

\section{B. Magnetoelastic coupling}

$\mathrm{NiO}$ has a lattice mismatch of $+0.5 \%$ when compared to $\mathrm{Fe}_{3} \mathrm{O}_{4}$. This means that a $\mathrm{NiO}$ layer grown on magnetite will be expanded on the interface plane and compressed along the surface normal by the epitaxial strain-if no strain relaxation via dislocations occurs. For the different interface orientations, the directions of the strain are varied with respect to the crystal lattice. Thus orientation-dependent magnetoelastic effects should occur. To discuss those, we estimate the layer distortions along the possible stacking directions ( $T$ domains ${ }^{18,49,50}$ ) in $\mathrm{NiO}$ by means of elastic continuum theory (see, for example, Ref. 51). For the (111) interface, the NiO layer is compressed along the surface normal [111] by about $0.24 \%$. Since the natural exchange striction in $\mathrm{NiO}$ also leads to a compression of $0.15 \%$ along the $\langle 111\rangle$-type stacking vectors,${ }^{52} \mathrm{NiO}$ should favor $T$ domains with a stacking vector normal to the surface. ${ }^{53}$ Consequently, the $\mathrm{NiO}$ easy plane will be coplanar with the (111) interface and a fairly low in-plane anisotropy should result. Indeed, Kurosawa et al. ${ }^{54}$ reported an extremely low value for well-annealed single crystals of only $29.5 \mathrm{~J} \mathrm{~m}^{-3}$. Our experimental situation, however, may be more compatible with a layer coupled to a single crystal as, e.g., reported by Lai et al. ${ }^{4}$ for $\mathrm{NiO}(111) /$ $\mathrm{NiFe}$. In this case, a considerably higher value of $1360 \mathrm{~J} \mathrm{~m}^{-3}$ was found for the $\mathrm{NiO}$ anisotropy within the (111) plane, which might be more realistic due to the interaction with the coupled layer. Lai et al. observed that the tensile epitaxial strain by the Permalloy orients the Ni surface moments in plane, which is quite close to our situation.

It is also important to note that the anisotropy of magnetite within the (111) plane has a value of only about $302 \mathrm{~J} \mathrm{~m}^{-3}$ and is thus comparable to $\mathrm{NiO}$ or even lower. ${ }^{55}$ Thus, we may envisage that the $\mathrm{Fe}_{3} \mathrm{O}_{4} / \mathrm{NiO}$ interface is topographically compensated, partly compensating the topmost magnetite layer as well according to the following picture. Ferromagnetic sheets of $\mathrm{NiO}$ couple locally parallel to the net moment of $\mathrm{Fe}_{3} \mathrm{O}_{4}$. Thus, the interface is microscopically (on the length scale of the lattice parameter) uncompensated with mesoscopic antiphase boundaries due to interface terraces (with dimensions on the order of tens to hundreds of lattice constants). As a consequence, a microscopic parallel coupling should result but with a macroscopically compensated magnetization due to the mesoscopic antiphase domains. Of course, in addition to these frozen spins there is another class of magnetic moments with a low anisotropy. ${ }^{56,57}$ Interfacial spins inside the antiphase boundaries are able to rotate more freely due to their frustrated coupling environment and may align parallel to the net magnetization in the substrate. The same mechanism accounts for defect spins. In this way, a small uncompensated magnetization in $\mathrm{NiO}$ is retained.

In case of the (110) interface, in-plane expansion of $0.5 \%$ renders the in-plane [111]- and [111]-stacking directions unfavorable (Fig. 9). Stacking along those axes would lead to spin-flop coupling, since the easy planes of $\mathrm{NiO}$ would be perpendicular to the interface plane, leaving as only intersec-
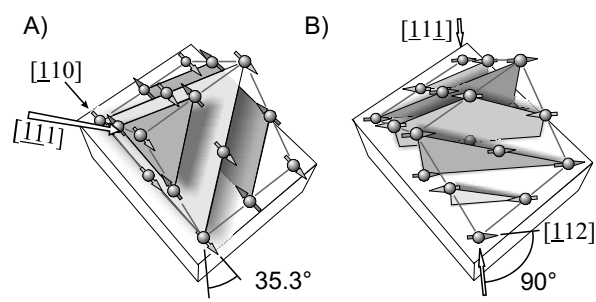

FIG. 9. Magnetoelastic situation at the (110) interface. (A) The tensile in-plane strain leads to an out-of-plane compression, which favors stacking along out-of-plane $\langle 111\rangle$ directions. The in-plane easy axis in this case is along [110]. (B) If the stacking were to occur in plane along the magnetite easy axis, the easy planes would be perpendicular to that axis, leading to spin-flop coupling. Since, however, the in-plane stain is tensile and moreover the magnetostriction in magnetite is positive along its easy axis, this situation is highly unlikely.

tions the in-plane $[ \pm 1 \mp 12]$ easy directions (compare Fig. 9(B)). Consequently, spin-flop coupling must be considered unlikely. The out-of-plane [11 1 1] directions, however, are favored by the out-of-plane compression (for example, 1.25 $\times 10^{-4}$ along [111]). Thus, the $\mathrm{NiO}$ stacking will take place along these axes and the $\mathrm{NiO}$ easy planes form an angle of $35.27^{\circ}$ with the interface (Fig. 9(A)). The intersection axis is then $[110]$, which forms a smaller angle with the $[ \pm 1 \mp 11]$ than with the $[ \pm 1 \mp 12]$ axes. This means that the coupling of $\mathrm{NiO}$ will be basically collinear with $\mathrm{Fe}_{3} \mathrm{O}_{4}$ but with a contribution of a uniaxial anisotropy along [110]. This situation is comparable to noncollinear exchange bias, where the easy axes of the $\mathrm{F}$ and AF differ. ${ }^{58}$ While in our as-grown samples we could not find a noticeable deviation from the $[ \pm 1 \mp 11]$ easy directions in the substrate, annealing of the layers induces a sizable deviation of the easy directions toward [110], giving additional support to our assumption. ${ }^{42}$

Finally, we will discuss the situation at the (001) interface, where all $\langle 111\rangle$-stacking directions are affected equally by the lattice strain (expansion by about $0.2 \%$ ). In this particular case, weaker magnetoelastic effects can come into play, such as, for example, magnetostriction. From our data we know that spin-flop coupling is realized, in striking contrast to the other interfaces. If we consider that the easy directions at the $\mathrm{Fe}_{3} \mathrm{O}_{4}(001)$ surface are in plane along the $\langle 110\rangle$ directions, we can estimate the magnetoelastic deformation at the interface from the magnetostriction constants of $\mathrm{Fe}_{3} \mathrm{O}_{4}{ }^{59}$ $\mathrm{NiFe}_{2} \mathrm{O}_{4},{ }^{60}$ and $\mathrm{NiO} .{ }^{61}$ Since the easy directions of bulk magnetite are $\langle 111\rangle$, the in-plane projections in the surface domains will be along $\langle 110\rangle$. In Table II we summarize the distortions along the [110] substrate magnetization direction

TABLE II. Magnetoelastically induced distortions in the $\mathrm{Fe}_{3} \mathrm{O}_{4}(001) / \mathrm{NiO}$ system.

\begin{tabular}{lcc}
\hline \hline Material & {$[110] \times 10^{-5}$} & {$[\underline{110}] \times 10^{-5}$} \\
\hline $\mathrm{Fe}_{3} \mathrm{O}_{4}(\mathbf{S} \|[110])$ & +5.5 & -6.5 \\
$\mathrm{NiFe}_{2} \mathrm{O}_{4}(\mathbf{S} \|[110])$ & -2.8 & +0.5 \\
$\mathrm{NiO}(\mathbf{S} \|[110]$, collinear $)$ & -9.4 & +0.2 \\
$\mathrm{NiO}(\mathbf{S} \|[110]$, spin flop $)$ & +0.2 & -9.4 \\
\hline \hline
\end{tabular}




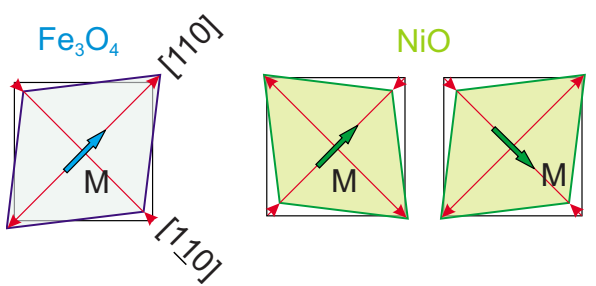

FIG. 10. (Color online) Magnetoelastic deformations of $\mathrm{NiO}$ and magnetite within the (001)-interface plane (situation exaggerated by a factor of $\left.2 \times 10^{3}\right)$. Using the magnetostriction constants of $\mathrm{NiO}$ (Ref. 61) and $\mathrm{Fe}_{3} \mathrm{O}_{4}$ (Ref. 59), one obtains that the deformations in both materials match only for spin-flop coupling.

and perpendicular to it $([\underline{110]})$ for the two cases of collinear and spin-flop coupling of NiO. As is directly apparent, the signs of the distortions in $\mathrm{NiO}$ along [110] and [-110] for spin-flop coupling match with the respective signs of magnetite, while the signs for collinear coupling are inverted (Fig. 10). Thus, spin-flop coupling of $\mathrm{NiO}$ is strain compatible with the magnetoelastic deformation of $\mathrm{Fe}_{3} \mathrm{O}_{4}$, while collinear coupling is not.

Although the strain due to magnetostriction is small (on the order of $10^{-5}$ ), we calculate a considerable energy gain of the spin-flop state as compared to that of the collinear state of $\Delta E_{\mathrm{MS}} \approx 3560 \mathrm{~J} \mathrm{~m}^{-3}$. For this estimation we assumed that the NiO layer simply follows the magnetostrictive strain of the substrate. We first calculated the elastic deformation of magnetite for $\mathbf{S} \|[110]$ and then used the magnetostriction constants for $\mathrm{NiO}$ from Ref. 61 together with the strain tensor induced by the substrate to calculate the magnetoelastic energy (see, for example, Ref. 51). Comparing the values for $\mathbf{S} \|[110]$ (collinear) and $\mathbf{S} \|[110]$ (spin flop), the magnetoelastic energy gain by spin-flop coupling is thus on the same order as other anisotropies in the sample. [For comparison, see the anisotropy on the (111) plane of $\mathrm{NiO}$ and magnetite as given earlier in this section.]

In order to judge whether it is possible that this energy overcomes the exchange coupling, we estimate the total magnetoelastic energy in our film in relation to the interfacial exchange energy at the $\mathrm{Fe}_{3} \mathrm{O}_{4}(001) / \mathrm{NiO}$ interface. Taking the strongest $\mathrm{Ni}-\mathrm{Fe}$ exchange constant in NFO, namely, the $124^{\circ}$ superexchange of $J_{A B}=-25 k_{B},{ }^{62}$ we can estimate the interfacial exchange energy as $E_{\mathrm{ex}} \approx 8 \times 10^{-3} \mathrm{~J} \mathrm{~m}^{-2}$ in the case of completely parallel coupling (uncompensated interface). This value is well within the range for $\mathrm{NiO}(100)$ as reported in Ref. 2. If the interface is partially compensated, however, this value will be further reduced. In the extreme case of a full compensation, it is smaller by approximately $\sin (\gamma), \gamma$ being the canting angle. As this angle is small-on the order of $5^{\circ}-10^{\circ}$ as found in our experiments-the interfacial exchange energy can be as low as $10^{-4} \mathrm{~J} \mathrm{~m}^{-2}$. On the other hand, if we estimate the total magnetoelastic energy in our thin film (thickness, $43 \AA$ ), we arrive at an energy of $\approx 1.6 \times 10^{-5} \mathrm{~J} \mathrm{~m}^{-2}$, which is 1 to 2 orders of magnitude lower than the estimated exchange energy. Taking into account that the interfacial exchange and magnetoelastic interactions were estimated from bulk values which may vary considerably at the interface, we believe that it is possible that both energies have indeed the same order of magnitude.
In this context, it should also be pointed out that, due to the long-ranged nature of the magnetoelastic strain fields, the total magnetoelastic energy gain increases with the volume, i.e., the layer thickness, while the interfacial exchange energy is constant. Hence, a threshold thickness exists at which the magnetoelastic energy overcomes the interfacial exchange energy and determines the coupling if exchange and magneoelasticity favor different coupling geometries.

Expanding on our findings, we actually propose that spin reorientation transitions observed in other studies, ${ }^{11,34}$ especially for $\mathrm{Fe}(001) / \mathrm{NiO},{ }^{11}$ can eventually be reinterpreted in this manner, too. Finazzi et al. ${ }^{11}$ observed a switching of the spin-axis orientation from spin flop to collinear in $\mathrm{Fe}(001) / \mathrm{NiO}\left(R 45^{\circ}\right)$ above a $\mathrm{NiO}$ thickness of about $20 \AA$. However they interpreted those results exclusively by an increase in defects with thickness. The reorientation, however, could also be explained by strain relaxation effects: At low thicknesses, strain leads to spin-flop coupling. Note that the situation is quite similar to ours because the magnetostriction in bcc iron is positive along the iron [100] axis, ${ }^{63}$ which is the [110] axis in $\mathrm{NiO}$. Thus, the $\mathrm{NiO}$ film is expanded along the substrate magnetization direction, just as in $\mathrm{Fe}_{3} \mathrm{O}_{4}(001) / \mathrm{NiO}$. Assuming that at some higher thickness strain relaxation occurs, the spin-axis orientation will switch back to collinear, if, for example, some uncompensated magnetization is mediated via defects (see results of Finazzi et $\left.a l .{ }^{11}\right)$. We further mention that the hypothesis of strain relaxation effects was also invoked by Krishnakumar et al. ${ }^{34}$ for $\mathrm{Ag}(001) / \mathrm{NiO} / \mathrm{MgO}$ systems.

From this discussion we may conclude that magnetoelastic effects can indeed lead to spin-flop coupling at the (001) interface if the magnetoelastic energy gain dominates over interfacial exchange coupling. Finally, we note that for $\mathrm{NiFe}_{2} \mathrm{O}_{4}$ (NFO) coupled to magnetite, the situation would be the same as for $\mathrm{NiO}$ - so spin-flop coupling could result even for a NFO-type reconstructed zone at the interface.

\section{Amount of uncompensated magnetization}

While the (111) and (001) interfaces yield a comparably low value for the uncompensated magnetization in $\mathrm{NiO}$, the (110) interface shows a considerably higher value. There may be several reasons for this behavior. First, the (110) interface is the only one which has easy axes of the substrate lying in the plane. This means that the in-plane anisotropy is larger than for the other orientations, stabilizing the $\mathrm{Ni}$ interface moments, which are in turn exchange coupled to the substrate. Second, interfacial bonding may affect the stability of the uncompensated magnetization, too. At the (110) interface, the bonding anisotropy is the largest among the three interfaces because the rotation symmetry about the surface normal is only $C_{2}$, whereas at (111) it is $C_{3}$ and $C_{4}$ for (001).

Furthermore, while at the (110) interface, two bonds connect a $\mathrm{Ni}$ atom to the underlying ferrimagnet at an angle of $45^{\circ}$ to the plane, at the (001) interface, there is only one bond oriented perpendicular to the surface. As a consequence, the exchange coupling and thus the amount of uncompensated magnetization should be lower for the (001) orientation than for the (110) case. At the (111) interface, there should be 
three bonds with out-of-plane components, so one would expect the induced moment to be stronger than in the other two cases. However, as discussed in Sec. V B, the stacking is out of plane in $\mathrm{NiO}$, leading to a topographic antiphase domain pattern in $\mathrm{NiO}$, with a macroscopic compensation of the interface magnetization. The residual Ni-XMCD signal might be caused by frustrated spins inside the antiphase boundaries or defect spins. Additionally, the in-plane anisotropy of $\mathrm{NiO}(111)$ is very low.

Last but not the least, the third relevant factor is a possible reconstruction of the interface after deposition of the antiferromagnet. While the data for the (110) interface allow conclusions about the interfacial phase and thus its structure, this is not possible for the other two interfaces due to the lack of thickness-dependent measurements. We thus cannot exclude that the absence of an interfacial $\mathrm{NiFe}_{2} \mathrm{O}_{4}$ phase in the (001) and (111) cases is responsible for the drastically reduced $\mathrm{Ni}$ XMCD signal.

\section{SUMMARY AND CONCLUSION}

In summary, we have investigated the role of the crystalline orientation of the FIM/AF interface in the magnetic proximity effect for the three low-indexed orientations (001), (110), and (111). Exploiting XMCD to map the substrate magnetization as well as anisotropic XMLD to extract the $\mathrm{NiO}$ spin-axis orientation, we found collinear coupling between the FIM and the AF for the (111) and the (110) interfaces, while the (001) interface exhibits spin-flop coupling.
In all three orientations, we observed an uncompensated magnetization in the AF near the interface by Ni XMCD. A comparison of the size of this magnetization contribution yields the largest value for the (110) interface, while the (111) and (001) interfaces exhibit only $10 \%$ of that value. The coupling type can be consistently explained for all three orientations by a strain-induced AF stacking asymmetry in the NiO AF domain pattern. For the (111) and (110) orientations, out-of-plane stacking leads to collinear coupling, while for the (001) orientation, the epitaxial strain does not prefer any particular stacking vector. In this case, magnetostrictive deformations induced by the magnetite substrate would favor spin-flop coupling, while the exchange coupling prefers collinear coupling. As a consequence, the system is subject to a delicate balance of several spin-dependent interactions. In this situation, a weak interfacial exchange coupling may thus lead to a spin-flop coupling via magnetoelastic effects.

\section{ACKNOWLEDGMENTS}

This investigation was performed in part at the beamline UE 56/1 SGM, BESSY GmbH Berlin, as well as at the SIM beamline, Swiss Light Source, Paul Scherrer Intitute, Switzerland. We thank Y.-X. Su and D. Schrupp for supplying the synthetic magnetite crystals and M. Schmidt and C. Thomas for the Laue measurements. Financial support through the Deutsche Forschungsgemeinschaft within SFB 491 is gratefully acknowledged. We commemorate F. U. Hillebrecht who initiated this work, but passed away unexpectedly in 2006. *i.krug@fz-juelich.de

†Deceased.

¥Present address: Max-Planck-Institut für Festkörperforschung, 70569 Stuttgart, Germany.

${ }^{1}$ W. Meiklejohn and C. Bean, Phys. Rev. 102, 1413 (1956).

${ }^{2}$ J. Nogues and I. K. Schuller, J. Magn. Magn. Mater. 192, 203 (1999).

${ }^{3}$ R. Coehoorn, in Handbook of Magnetic Materials, edited by K. Buschow (Elsevier Science, Amsterdam, 2003), Vol. 15, p. 4.

${ }^{4}$ C. H. Lai, T. Ming, R. Erwin, and J. Borchers, J. Appl. Phys. 91, 7751 (2002).

${ }^{5}$ A. Barbier, C. Mocuta, W. Neubeck, M. Mulazzi, F. Yakhou, K. Chesnel, A. Sollier, C. Vettier, and F. de Bergevin, Phys. Rev. Lett. 93, 257208 (2004).

${ }^{6}$ C. Tusche, H. L. Meyerheim, F. U. Hillebrecht, and J. Kirschner, Phys. Rev. B 73, 125401 (2006).

${ }^{7}$ S. Roy et al., Phys. Rev. Lett. 95, 047201 (2005).

${ }^{8}$ F. U. Hillebrecht, H. Ohldag, N. B. Weber, C. Bethke, U. Mick, M. Weiss, and J. Bahrdt, Phys. Rev. Lett. 86, 3419 (2001).

${ }^{9}$ H. Ohldag, A. Scholl, F. Nolting, S. Anders, F. U. Hillebrecht, and J. Stohr, Phys. Rev. Lett. 86, 2878 (2001).

${ }^{10}$ H. Ohldag, A. Scholl, F. Nolting, E. Arenholz, S. Maat, A. T. Young, M. Carey, and J. Stohr, Phys. Rev. Lett. 91, 017203 (2003).

${ }^{11}$ M. Finazzi, A. Brambilla, P. Biagioni, J. Graf, G.-H. Gweon, A. Scholl, A. Lanzara, and L. Duo, Phys. Rev. Lett. 97, 097202
(2006).

${ }^{12}$ I. P. Krug, F. U. Hillebrecht, M. W. Haverkort, H. Gomonay, A. Tanaka, L. H. Tjeng, and C. M. Schneider, Europhys. Lett. 81, 17005 (2008).

${ }^{13}$ M. Kiwi, Origin of the Magnetic Proximity Effect, MRS Symposia Proceedings No. 746 (Materials Research Society, Pittsburgh, 2003), p. Q.5.2.1.

${ }^{14}$ M. W. Haverkort, S. I. Csiszar, Z. Hu, S. Altieri, A. Tanaka, H. H. Hsieh, H. J. Lin, C. T. Chen, T. Hibma, and L. H. Tjeng, Phys. Rev. B 69, 020408(R) (2004).

${ }^{15}$ D. Alders, L. H. Tjeng, F. C. Voogt, T. Hibma, G. A. Sawatzky, C. T. Chen, J. Vogel, M. Sacchi, and S. Iacobucci, Phys. Rev. B 57, 11623 (1998).

${ }^{16}$ M. Finazzi and S. Altieri, Phys. Rev. B 68, 054420 (2003).

${ }^{17}$ S. I. Csiszar, M. W. Haverkort, Z. Hu, A. Tanaka, H. H. Hsieh, H. J. Lin, C. T. Chen, T. Hibma, and L. H. Tjeng, Phys. Rev. Lett. 95, 187205 (2005).

${ }^{18}$ T. Yamada, J. Phys. Soc. Jpn. 21, 664 (1966).

${ }^{19}$ T. J. Moran, J. Nogués, D. Lederman, and I. K. Schuller, Appl. Phys. Lett. 72, 617 (1998).

${ }^{20}$ J. Nogues, T. J. Moran, D. Lederman, I. K. Schuller, and K. V. Rao, Phys. Rev. B 59, 6984 (1999).

${ }^{21}$ X. Qian and W. Hubner, Phys. Rev. B 67, 184414 (2003).

${ }^{22}$ N. C. Koon, Phys. Rev. Lett. 78, 4865 (1997).

${ }^{23}$ P. J. van der Zaag, A. R. Ball, L. F. Feiner, R. M. Wolf, and P. A. A. van der Heijden, J. Appl. Phys. 79, 5103 (1996). 
${ }^{24}$ D. Sander, A. Enders, and J. Kirschner, J. Magn. Magn. Mater. 200, 439 (1999).

${ }^{25}$ A. Recnik, D. L. Carroll, K. A. Shaw, D. M. Lind, and M. Ruhle, J. Mater. Res. 12, 2143 (1997).

${ }^{26}$ H. Q. Wang, W. Gao, E. I. Altman, and V. E. Henrich, J. Vac. Sci. Technol. A 22, 1675 (2004).

${ }^{27}$ T. J. Regan, H. Ohldag, C. Stamm, F. Nolting, J. Luning, J. Stohr, and R. L. White, Phys. Rev. B 64, 214422 (2001).

${ }^{28}$ M. Finazzi, M. Portalupi, A. Brambilla, L. Duo, G. Ghiringhelli, F. Parmigiani, M. Zacchigna, M. Zangrando, and F. Ciccacci, Phys. Rev. B 69, 014410 (2004).

${ }^{29}$ J. Kuneš and P. M. Oppeneer, Phys. Rev. B 67, 024431 (2003).

${ }^{30}$ J. Kuneš, P. M. Oppeneer, S. Valencia, D. Abramsohn, H.-C. Mertins, W. Gudat, M. Hecker, and C. M. Schneider, J. Magn. Magn. Mater. 272-276, 2146 (2004).

${ }^{31}$ S. Czekaj, F. Nolting, L. J. Heyderman, P. R. Willmott, and G. van der Laan, Phys. Rev. B 73, 020401(R) (2006).

${ }^{32}$ E. Arenholz, G. van der Laan, R. V. Chopdekar, and Y. Suzuki, Phys. Rev. B 74, 094407 (2006).

${ }^{33}$ E. Arenholz, G. van der Laan, R. V. Chopdekar, and Y. Suzuki, Phys. Rev. Lett. 98, 197201 (2007).

${ }^{34}$ S. Krishnakumar, M. Liberati, C. Grazioli, M. Veronese, S. Turchini, P. Luches, S. Valeri, and C. Carbone, J. Magn. Magn. Mater. 310, 8 (2007).

${ }^{35}$ F. Schedin, E. W. Hill, G. van der Laan, and G. Thornton, J. Appl. Phys. 96, 1165 (2004).

${ }^{36}$ P. J. Jensen, H. Dreyssé, and M. Kiwi, Eur. Phys. J. B 46, 541 (2005).

${ }^{37}$ S. Murphy, S. F. Ceballos, G. Mariotto, N. Berdunov, K. Jordan, I. V. Shvets, and Y. M. Mukovskii, Microsc. Res. Tech. 66, 85 (2005)

${ }^{38}$ E. Goering, S. Gold, M. Lafkioti, and G. Schutz, Europhys. Lett. 73, 97 (2006).

${ }^{39}$ M. Pilard, O. Ersen, S. Cherifi, B. Carvello, L. Roiban, B. Muller, F. Scheurer, L. Ranno, and C. Boeglin, Phys. Rev. B 76, 214436 (2007).

${ }^{40}$ H. Ohldag, Ph.D. thesis, Düsseldorf, 2003.

${ }^{41}$ Note that the same plot may also account for collinear coupling of $\mathrm{NiO}$ by interchanging the two labels $\mathrm{D} 1$ and $\mathrm{D} 2$ for the $\mathrm{NiO}$ contrast.

${ }^{42}$ I. P. Krug, Ph.D. thesis, Jülich/Duisburg, 2008.
${ }^{43}$ A. Scholl, M. Liberati, E. Arenholz, H. Ohldag, and J. Stohr, Phys. Rev. Lett. 92, 247201 (2004).

${ }^{44}$ The scatter of the data points is thus due to (nonmagnetic) intensity fluctuations possibly caused by an imperfect undulator control. A normalization to the primary intensity $I_{0}$ measured at the refocusing mirror could not eliminate the scatter.

${ }^{45}$ J. A. Borchers, Y. Ijiri, D. M. Lind, P. G. Ivanov, R. W. Erwin, S. H. Lee, and C. F. Majkrzak, J. Appl. Phys. 85, 5883 (1999).

${ }^{46}$ J. A. Borchers, Y. Ijiri, D. M. Lind, P. G. Ivanov, R. W. Erwin, A. Qasba, S. Lee, K. V. O’Donovan, and D. C. Dender, Appl. Phys. Lett. 77, 4187 (2000).

${ }^{47}$ Since the anisotropy on the (111) plane is very low, there are practically no extended regions of homogeneous magnetization but rather a continuous modulation of the magnetization vector along the surface.

${ }^{48}$ For the electron escape depth in $\mathrm{NiO}, \lambda_{e}=30 \AA$ was assumed (Ref. 27). The x-ray-absorption length was determined to be $555 \AA$ at the energy of $855.8 \mathrm{eV}$.

${ }^{49}$ W. L. Roth, J. Appl. Phys. 31, 2000 (1960).

${ }^{50}$ T. Yamada, S. Saito, and Y. Shimomura, J. Phys. Soc. Jpn. 21, 672 (1966).

${ }^{51}$ D. Sander, Rep. Prog. Phys. 62, 809 (1999).

${ }^{52}$ G. A. Slack, J. Appl. Phys. 31, 1571 (1960).

${ }^{53}$ T. Yamada, J. Phys. Soc. Jpn. 18, 520 (1963).

${ }^{54}$ K. Kurosawa, M. Miura, and S. Saito, J. Phys. C 13, 1521 (1980).

${ }^{55}$ O. Ozdemir, S. Xu, and D. J. Dunlop, J. Geophys. Res., [Solid Earth] 100, 2193 (1995).

${ }^{56}$ F. Radu, A. Nefedov, J. Grabis, G. Nowak, A. Bergmann, and H. Zabel, J. Magn. Magn. Mater. 300, 206 (2006).

${ }^{57}$ H. Ohldag, H. Shi, E. Arenholz, J. Stohr, and D. Lederman, Phys. Rev. Lett. 96, 027203 (2006).

${ }^{58}$ S. P. Pogossian, D. Spenato, D. T. Dekadjevi, and J. B. Youssef, Phys. Rev. B 73, 174414 (2006).

${ }^{59}$ G. D. Klapel and P. N. Shive, J. Geophys. Res. 79, 2629 (1974).

${ }^{60}$ A. B. Smith and R. V. Jones, J. Appl. Phys. 37, 1001 (1966).

${ }^{61}$ T. G. Phillips and R. L. White, Phys. Rev. 153, 616 (1967).

${ }^{62}$ S. J. Kim, W. C. Kim, C. S. Kim, and S. W. Lee, J. Korean Phys. Soc. 36, 430 (2000).

${ }^{63}$ E. Arakawa, K. Maruyama, K. Mori, H. Nishigaitsu, and N. Aizawa, IEEE Trans. Magn. 41, 3718 (2005). 